\title{
Zonamiento mineralógico con difracción de rayos X para la optimización del tratamiento metalúrgico en el Yacimiento Toromocho
}

\author{
Mineralogical zoning with X-ray difraction for the optimization of metallurgical \\ treatment in the Toromocho Deposit
}

Ronald Eugenio Flores Barrón 1, Enrique Guadalupe Gómez ${ }^{2}$

Recibido: 01/07/2020 - Aprobado: 30/10/2020 - Publicado: 30/11/2020

\begin{abstract}
RESUMEN
El yacimiento Toromocho es un yacimiento pórfido skarn con una extensión de $5 \mathrm{~km}$ por $6 \mathrm{~km}$, geológicamente está constituida por rocas volcánicas del Mitu y calizas del Pucara e intrusivos terciario. La mineralización, mayormente está dada por vetas que atraviesan de Este a Oeste en todo Morococha que están rellenadas con minerales de $\mathrm{Pb}, \mathrm{Zn}$ y Ag mantos de Zinc emplazados en las calizas y en forma diseminada, también emplazados en las calizas y en los contactos volcánicos-caliza-intrusivo y también cuerpos piritosos diseminados con mineralización de cobre. En cuanto a su mineralización es muy compleja encontrando mantos, vetas, stockwork, diseminado cuerpos encontrando minerales que afectan mucho el tratamiento metalúrgico como es el arsénico. Los resultados de los estudios de XRD nos permite caracterizar los minerales existentes en el yacimiento, de los minerales contaminantes para poder realizar un buen blending, controlar el talco, fluor magnesio, zinc, etc. A mayor contenido de talco en CMC es más efectivo a un con gangas naturalmente flotables para lograr un grado mínimo de $20 \%$ requiere $700 \mathrm{~g} / \mathrm{l}$ de $\mathrm{CMC}$. Según acuerdo con el área de metalurgia, planeamiento y geología se llegaron a nos términos de no considerar algunos minerales penalizables como es el fluor, porque en la parte central del yacimiento se tiene alto fluor como también alto grado de cobre que esto permite realizar un blending para poder levantar la ley de cobre, pero en la planta metalúrgica se tiene que activar el proceso de la planta de molibdeno para poder limpiar el mineral con alto fluor y talco con la adición del $\mathrm{NaSH}$.
\end{abstract}

Palabras clave: Pórfido; mineralización; vetas; mantos; stockwork; blending.

\begin{abstract}
The Toromocho Deposit is a Porphyry Skarn deposit with an extension of $5 \mathrm{~km}$ by $6 \mathrm{~km}$, geologically it is made up of volcanic rocks from Mitú and limestone from Pucara and tertiary intrusives. The mineralization is mainly due to veins that cross the entire Morococha district from East to West, which are filled with $\mathrm{Pb}, \mathrm{Zn}$ and $\mathrm{Ag}$ Zinc mantles minerals located in the limestones and in a disseminated form, also located in the limestones and in the Volcanic-limestone-intrusive contacts and also scattered pyritic bodies with copper mineralization. Regarding its mineralization, it is very complex finding veins, mantles, stock work, scattered bodies, finding minerals that greatly affect metallurgical treatment such as arsenic. The results of the XRD studies allow us to characterize the existing minerals in the deposit, from the contaminating minerals to be able to perform a good blending, control talc, fluorine, magnesium, zinc, etc. The higher the talc content in $\mathrm{CMC}$, the more effective it is with naturally buoyant bargains to achieve a minimum degree of $20 \%$. It requires $700 \mathrm{~g} / \mathrm{I}$ of CMC. According to agreement with the metallurgy, planning and geology area, we came to terms of not considering some penalizable minerals such as fluorine, because in the central part of the deposit there is high fluorine as well as a high degree of copper, which allows blending. to be able to raise the copper grade, but in the metallurgical plant the molybdenum plant process has to be activated to be able to clean the mineral with high fluorine and talc with the addition of $\mathrm{NaSH}$.
\end{abstract}

Keywords: Porphyry; mineralization; veins; mantles; stock work; blending.

\footnotetext{
Universidad Nacional Mayor de San Marcos, Facultad de Ingeniería Geológica, Minera, Metalúrgica y Geográfica. Lima, Perú. Ingeniero. Maestría en Geología con mención en Minas y Recursos Energéticos. Autor para correspondencia: ronaldbarron05@hormail.com ORCID: https://orcid.org/0000-0003-3591-9503

2 Universidad Nacional Mayor de San Marcos, Facultad de Ingeniería Geológica, Minera, Metalúrgica y Geográfica. Lima, Perú. Magister. E-mail: eguadalupeg@unmsm.edu.pe ORCID: https://orcid.org/0000-0001-9583-8807
} 


\section{INTRODUCCIÓN}

El yacimiento de Toromocho está situado en Morococha - Provincia de Yauli, Departamento de Junín, en el Centro del Perú, la reorganización de la Compañía Morococha se dio en 1915, y se agrupó con Cerro de Pasco Corp. Con el paso de los años, 3 años para ser específicos, Backus \& Johnston transfirió su parte en Morococha a Cerro de Pasco Corp.; posteriormente, con la apropiación de Cerro de Pasco Corp., en 1974, Morococha se transformó en una entidad de elaboración bajo la dirección de Centromin.

La investigación originaria inscrita en la cantera de Toromocho nos lleva al año 28, en donde reveló un área mineralizada de cobre de baja ley a lo extenso del stock de granodiorita en el Cerro San Francisco.

Las investigaciones extensivas prosiguieron con cuatro (4) operaciones llevadas por Centromin y Cerro de Pasco Corp., comprendidos entre 1966 y 1976. Concluyeron 143 excavaciones, en estas campañas, con un total de 42,394 m. En agosto de 1980 Centromin empleó a Consultores MineroMetalúrgicos S.A y a Kaiser Ingenieros International Inc., para que realicen un estudio de perspectiva y ratificar, de esta manera, los valores de los almacenamientos y establecer lineamientos estratégicos y financieros.

En el 74, Centromin comenzó una utilización a cielo abierto a bajo nivel de la cantera de Toromocho. La representación más actual y completa de Morococha fue informada por Álvarez Angulo (1999).

En el 2003, la posesión fue desnacionalizada y cedida a MPCopper la cual en el 2003 realizó cinco (5) excavaciones semejantes a las de Centromin y estuvieron desarrolladas ratificando la presencia de una considerable cantera de cobre.

Incluso finales del 2008, MPCopper-MCP ha realizado en general 310 perforaciones diamantinos con un resultado de $126,537 \mathrm{~m}$, en excavaciones ligeras y bajo tierra.

Toromocho en un yacimiento de cielo abierto con almacenamientos de molibdeno y cobre, situada en los Andes del Perú (parte central); en Morococha (ver Figura 1), en una extensión que tiene una basta tradición de exploraciones mineras y que ha sido activamente investigada a partir de 1960 por Cerro de Pasco Corp.,

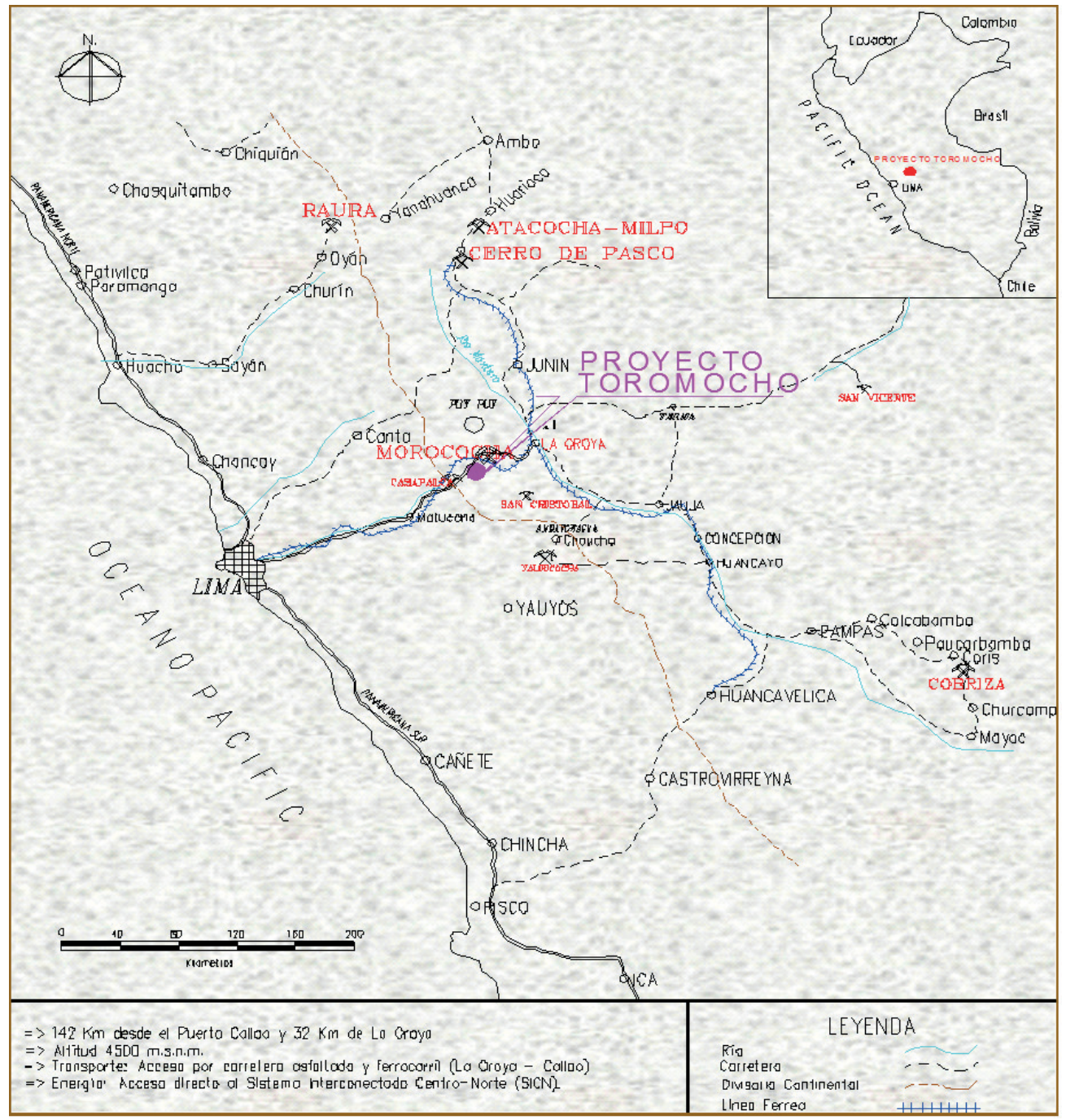

Figura1. Localización Proyecto Toromocho

Fuente: Minera Chinalco Perú S.A. (2020). 
después por Centromin y actualmente por adjudicación de Minera Chinalco Perú S.A. (2020).

\section{MÉTODOS}

Se realizó una investigación aplicada que buscará resolver problemas prácticos de mineralogía y su tratamiento por la naturaleza de los datos cuantitativos con un tratamiento de datos, que también es cualitativo. Además, en el laboratorio de XRD se realizó una interpretación de los problemas de mineralogía y tratamiento por el lugar de investigación analizando los diversos minerales; mientras, en el campo se ha hecho el zonamiento y recojo de muestras, que según la propuesta es estudio de observación de campo y de laboratorio.

La unidad de análisis fue el yacimiento Toromocho, con una población de estudio de 11000 muestras. El tamaño de la muestra fue de 8600 , cuyo criterio consistió en contrastar los resultados de laboratorio con datos de campo, por tener resultados anómalos.

\subsection{Técnicas de recolección de datos}

- Para este tipo de investigación se utilizaron muestras de blast hole para luego de ser ensayado para sus diferentes ensayos químicos.

- Para los análisis de XRD se utilizó pulpas de muestras para luego ser mezcladas o homogenizadas, luego se selecciona una muestra representativa del cuarteo unos 2 gramos de muestra para luego ser llevadas a la plaqueta.

- Se realizaron mapeos geológicos para poder correlacionar los resultados con la geología local.

- Se realizó mapeo de alteraciones de igual manera correlacionar los resultados con las alteraciones locales.

- Se recopiló lainformación disponible (geología y estudios preliminares).

\subsection{Análisis e interpretación de la información}

Para la interpretación se codifican las muestras una vez interpretadas en el XRD, para luego hacer una interpretación con el mapeo geológico y de alteraciones bien detalladas para luego realizar un blending adecuado con la cantidad litológicas, alteraciones y con las restricciones de los minerales contaminantes, también las datas nos sirvan para poder realizar un modelo de las UGM (Unidades Geo Metalúrgicas) y tener más detallado el blending, para un mejor performance metalúrgico.

Podemos encontrar altos valores de talco depositados a un stock y a la vez volver a manipularlos por contener altas leyes de cobre promedio de $0.70 \%$, pero tiene minerales insolubles de altos valores de fluor en promedio de 1532 ppm y talco $21 \%$ que causaron problemas espumándose en la totalidad de los espesadores trayendo problemas en la recuperación muy bajas, por no contar con una planta de pre-flotación de cobre donde se deprime el talco y flota el cobre, a raíz de estos problemas se comenzó a realizar un mapeo más detallado en las rocas sedimentarias y tipo de alteración para poder tener bien diferenciado en los taludes cuando se comienza a minar dicha banqueta, y con lo que respecta a los logueos de blast hole se trataba de sacar un promedio de porcentaje de talco, al tacto para poder colocarlo en las diferentes blast hole donde contenía skarn de serpentina- clorita magnetita con alteraciones de talco para luego interpretar.

Conforme se tenía los datos inciertos se adquirió un equipo de XRD con lecturas de 90 muestras al día de pulpas de mineral para poder cuantificar los contenidos de los minerales de la roca con mayor exactitud los componentes de los minerales insolubles (talco, fluor, magnesio etc.), se comenzó a realizar una malla para poder extraer las muestras de blasth hole y así poder enviar al laboratorio de XRD y saber las caracterización de dichas muestras y tener una visión de donde están los minerales insolubles y poder correlacionar con los mapeos en campo y poder realizar un zonamiento general de los tipos de rocas que se encuentran en la mina tanto rocas intrusivas como rocas sedimentarias.

En reunión con las diferentes áreas como planeamiento, geología, metalurgia y comercialización se puso restricciones al envió el mineral hacia la planta concentrador y su posterior envió a China.

Donde el fluor no sobrepasa de $1000 \mathrm{ppm}$. magnesio $2 \%$, arsénico de $350 \mathrm{ppm}$ y zinc de $3 \%$, en base a estas restricciones se tenía que realizar los planes de minado de mensuales trimestrales y anules, y de igual con el horfels se tenía restricciones menores al 30\% debido a su alta dureza, esto implicaba mayor tiempo de molienda limitando el tonelaje procesado por el hecho de tener un fracturamiento en forma laminar afectando a las bombas Geho.

El talco tenía una restricción hasta el 6\%, oxido de cobre $<16 \%$, todas estas restricciones se cambiaron el año 2019 debido a que no se llegaba a los tonelajes y leyes estimadas.

Para este año no hay restricciones con el fluor que superaba hasta $5000 \mathrm{ppm}$ porque muy aparte de estar alto lo limites se tenía muy buenas leyes de cobre en promedio hasta $1.5 \%$ de cobre según los análisis de muestreos de los blast hole, todas estas leyes altas se encuentran en la zona central del yacimiento Toromocho.

Para poder limpiar el fluor alto proveniente de las operaciones, el área de metalurgia tiene que activar la planta de molibdeno NASH el cual se realiza una flotación inversa esto significa que el concentrado es el relave en el cual los minerales son insolubles como el talco flogopita y las micas verdes, y el relave final de esta etapa es el concentrado de cobre.

También se limita en envió del skarn de serpentina magnetita menor al $30 \%$ debido a la presencia de magnetita o también a la presencia de los mantos de sulfuro semi masivo o de magnetita y pirita, también entra a las restricciones el skarn de actinolita - tremolita $<$ al $40 \%$ debido que produce material fino esto implica que las arcillas se quedan pegados en los chuts de chancado y también en el molino Sag. 
El material duro que es la roca granodiorítica que tiene una dureza de 4 también, donde tiene baja ley de cobre pero buena recuperación, pero tiene su implicancia con el alto consumo de energía en el Throuput, cuando entra solo al molino Sag, pero está en función a una buena fragmentación, por ende es necesario realizar un blending con materiales suaves como es el caso del skarn de actinolita tremolita / skarn de serpentina magnetita, para no tener problemas en el proceso metalúrgico por eso se tiene bien diferenciado todo el yacimiento por durezas que va desde la dureza 2 hasta la dureza 4, todo este plan de minado con las restricciones del 2019 se tiene un buen performances en la planta metalúrgica con muy buenas recuperaciones que llegan hasta el $85 \%$ de recuperación y grado hasta $25 \%$ de cobre como se puede observar.

En la actualidad se realiza un plano de polígonos de mineral con sus respectivas leyes de cobre esto nos ayuda para tener en cuenta el envío de mineral hacía de acuerdo con el blending que se realiza diaria, luego para poder realizar una conciliación final del envió de mineral tanto de mina como de planta metalúrgica. Se realiza también planos litológicos con sus respectivos cortes semanales para tener en consideración el mineral alimentado a planta de acuerdo con el plan de minado día a día tanto las rocas sedimentarias como las rocas intrusivas.

Las muestras en el blending con una ley $0.83 \%$ de cobre con todas las características que requiere la planta concentradora para poder llegar a fin de año con el KPI de los cobres finos a mayor tonelaje y buena ley se obtiene mayor tonelaje en los cobres finos.

\subsection{Clima y Meteorología}

Sobre la determinación del clima de la franja se discurrieron y examinaron los valores originarios de diferentes observatorios regionales colocados aledaños al espacio de investigación y unas cuantas estaciones oportunas de Chinalco. El espacio de estudio muestra un clima abrupto característico de ambientes situados en las elevaciones como la serranía del País. Estas temperaturas están diferenciadas especialmente por ser seco y frío, y por mostrar dos períodos visiblemente definidas: el periodo de precipitaciones, proporcionado a la etapa percibido entre octubre y marzo; y la época seca, proporcionado a los demás períodos del año.

El componente más acreditado que precisa la temperatura en el espacio de investigación es su enfoque altitudinal (alrededor de $4500 \mathrm{~m}$ de altitud en promedio). Otros componentes tomados en cuenta fueron la latitud (comprendidos entre 11 y $12^{\circ}$ de latitud sur), y la continentalidad o enfoque en relación al mar. Los componentes actúan a modo indiscutible en la peculiaridad atmosférica significativa tales como la extensión calidad en periodos diarios como también anuales, los sistemas eólicos, así como en los paralelismos existentes de precipitación, evaporación y humedad, Minera Chinalco Perú S.A. Knight Piésold, E. I. A. (2009)

Las exploraciones del observatorio meteorológico de Tuctu sellan una temperatura promedio cada mes entre $4,0^{\circ} \mathrm{C}$ y $5,9^{\circ} \mathrm{C}$; sin una diferenciación cada año característico y con una temperatura media cada año de $5,0^{\circ} \mathrm{C}$. Esta reducida inestabilidad es una peculiaridad propia de estas latitudes. Mayo, julio, agosto y noviembre muestran los datos más elevados de temperatura; con máximas cada mes promedio ascendentes a $12^{\circ} \mathrm{C}$; las temporadas con las más bajas precipitaciones entre junio, julio y agosto; con medias pequeñas cada mes de $-2^{\circ} \mathrm{C}$ en promedio.

En la media de cada año de luminiscencia solar habitual para el tiempo de exploración es $4627 \mathrm{Wh} / \mathrm{m}^{2}$. La investigación señala el límite promedio máximo cada mes de la alcanzada diariamente de $5479 \mathrm{Wh} / \mathrm{m}^{2}$ en agosto y un mínimo de $3838 \mathrm{Wh} / \mathrm{m}^{2}$ en el tercer mes del año.

Los vientos de la franja muestran peculiaridades propias de sistemas eólicos de montaña y de valle (brisas específicas) con una conducta afín con las pendientes cálidas determinadas en la zona, que establecen la magnitud de las corrientes de las aglomeraciones de aire, y una conducta enérgicamente influenciado por la distribución topográficas.

La diferenciación de velocidad del viento durante las 24 horas está comprendida especialmente por las permutas de temperatura en el viento (enfriamiento y calentamiento agrupado a los picos de arraigo del sol), de tal forma, la conducta de este lineamiento se define por mostrar un nivel pequeño firme (velocidad promedio de $2,2 \mathrm{~m} / \mathrm{s}$ ) durante la amanecida y el inicio del día. A partir de las horas matinales influenciadas por el calentamiento del ambiente, la rapidez del aire comienza a aumentar hasta alcanzar el dato máximo $5,9 \mathrm{~m} / \mathrm{s}$ en horas de la tarde.

En relación a la orientación del viento, se tiene que ésta modifica elocuentemente a lo largo del día, fruto del ambiente de los vientos de la zona (vientos de valle y montaña). De esta manera, durante la amanecida y al inicio del día, las corrientes de aire muestran un flujo que procede de un rango extenso de orientaciones: originarios del estenoreste (ENE). Y del oeste (O) al Sur-Suroeste (SSO) desde etapas de la mañana, esta variabilidad se comprime, prevaleciendo las orientaciones este-noreste (ENE) y noreste $(\mathrm{NE})$, concordando con las corrientes aéreas más rápidos, hasta pasado el mediodía. Antes del crepúsculo, según los vientos reducen sucesivamente de magnitud, provienen del este-noreste (ENE) y del oeste $(\mathrm{O})$.

La humedad respectiva conseguida varía entre $54,4 \%$ y $74,8 \%$, como valores de la media en la época de lluvia y entre $43,9 \%$ y $68,5 \%$, como datos promedio durante la época seca. El dato de saturación media a lo largo del espacio de registro es de $62,2 \%$.

El registro típico de precipitaciones durante el año presenta a los dos primeros meses como el tiempo con más precipitaciones registrando datos entre 88,4 y 132,3 mm y a mediados del año como el tiempo más árido con datos entre 7,4 y 17,2 mm. Los datos de los aguaceros promedios anuales fueron de $782,0 \mathrm{~mm}$ en el observatorio Huascacocha; 850,9 mm en el observatorio Morococha; y 537,6 mm en el observatorio Pucará. Los datos al año promedio de evaporación se encuentran entre los $1180 \mathrm{~mm}$ y $1262 \mathrm{~mm}$. Minera Chinalco Perú S.A. - Piensa Mas Alla (2020) (Ver Figuras 2-4). 


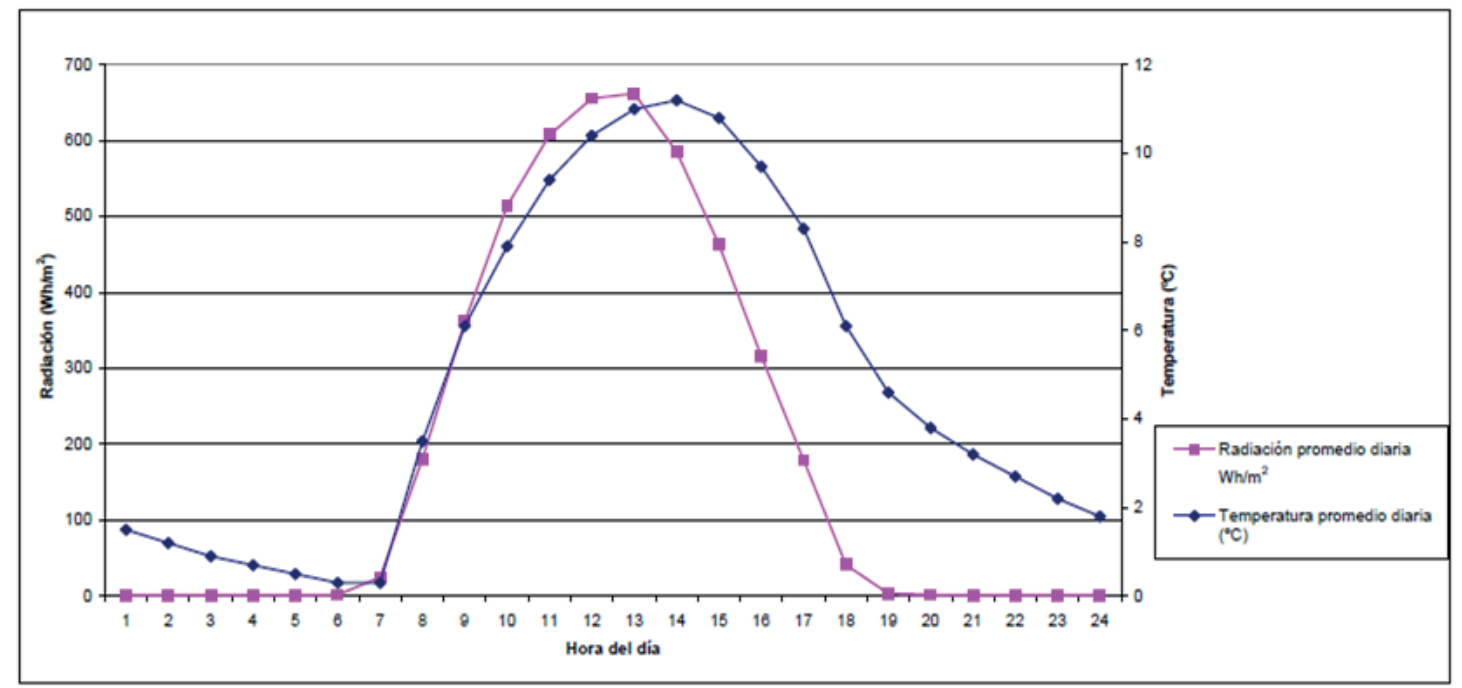

Figura 2. Variación de la Temperatura y Radiación Solar a lo largo del día - Estación Tuctu Fuente: Minera Chinalco Perú S.A. (2020)

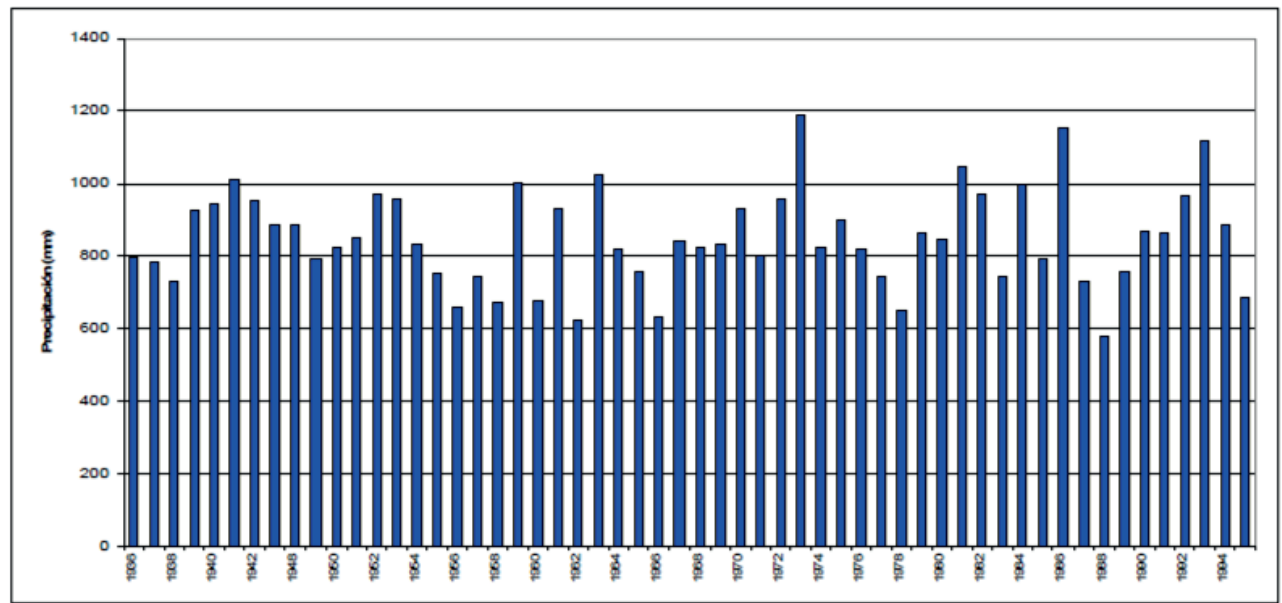

Figura 3. Precipitación Anual Morococha

Fuente: Minera Chinalco Perú S.A. (2020)

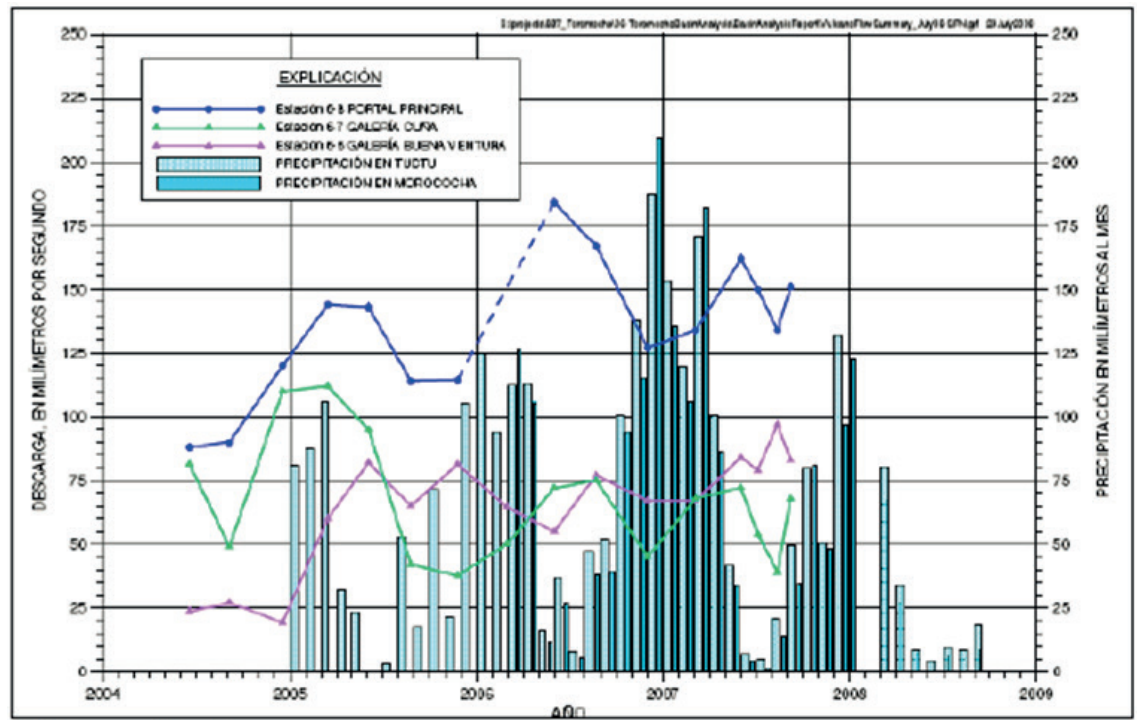

Figura 4. Línea de base hidrogeológica

Fuente: Montgomery \& Associates (2020) 


\subsection{Mineralogía}

Una basta diversidad de mena y minerales ganga se encontraron en Morococha, como se menciona:

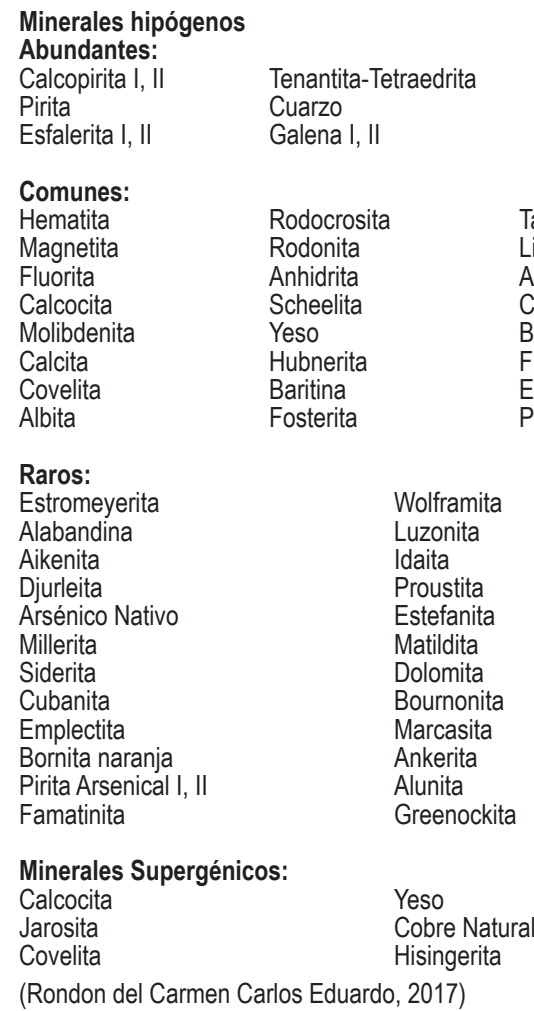

\subsection{Paragénesis y Zoneamiento del Distrito de Morococha}

En las primordiales etapas de la mineralización, molibdeno, plomo, cobre, zinc, y soluciones creadoras de plata, posiblemente descendientes del interior de los stocks de San Francisco y Gertrudis acometieron en Morococha almacenando vetas, mantos y otros insumos de materias inorgánicas reducidas, así como un considerable cuerpo de mineral céntrico disperso y de vetillas (stockwork) de Toromocho (Rondon del Carmen Carlos Eduardo, 2017).

La mineralización de cobre se reúne en el área céntrica, aledaño y adentro de los stocks de San Francisco y Gertrudis y en los entornos de los skarns y hornfels. Envolviendo a la zona céntrica de cobre y se encuentra en una franja media con mineralización de plomo como también de zinc y la parte externa que engloba los bordes de Morococha muestra mineralización de plata y plomo.

\subsection{Zonas de Mineralización}

\subsubsection{Escala del zoneamiento metálico del yacimiento}

El nivel de zoneamiento erguido de una cantera de molibdeno, arsénico y cobre, quizás además de niveles muy menores de oro, se halla en la cantera de Toromocho.

La Cantera de cobre se desarrolla hacia la parte inferior con un plano de base que se ubica entre 500 a 600 $\mathrm{m}$ inferior a la zona. La ley más elevada y primordial de la cantera mineral se encuentra adentrada en una cantera de 1.0 a $2.0 \mathrm{~km}$ de skarn enérgicamente fracturado envolviendo por los intrusivos granodioríticos y el pórfido feldespático con la apariencia de un domo que se considera con una antigüedad de 6 a 7 millones de años y es inferior a un intrusivo andesítico/diorítico regional más primitiva mostrada en el área (Ver Figura 5).

Los modelos de la distribución de molibdeno en la fragmento importante y central de la cantera expuestos en secciones tienen la representación de conoides invertidos o sección 27 oeste de cobre

El modelo del cono invertido extendido en unidades refleja la cantidad de vetillas tipo "B" de cuarzo - Mo

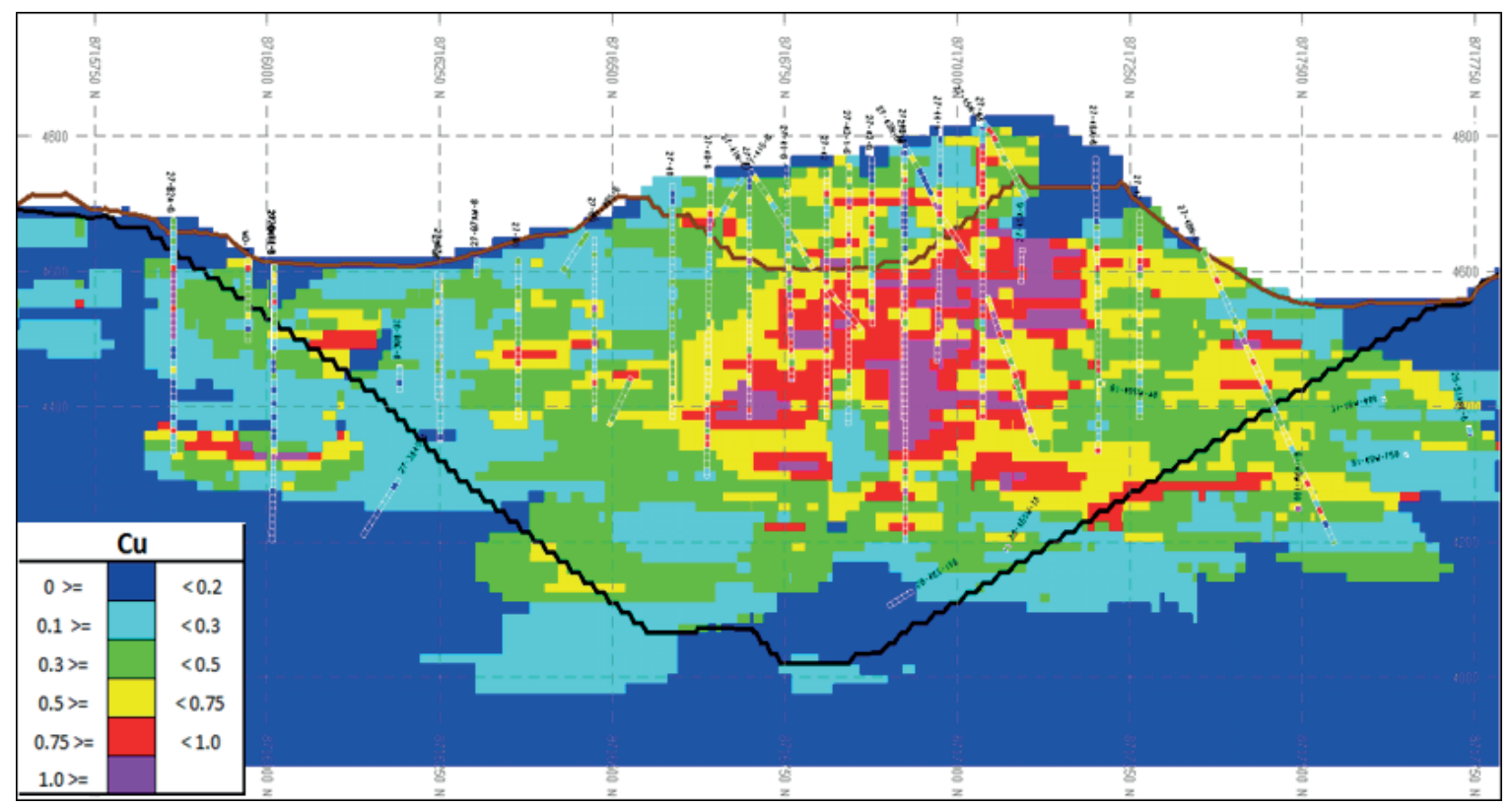

Figura 5. Sección $27 \mathrm{~W}$ Cu

Fuente: Minera Chinalco Perú S.A. (2020) 
en la mineralización prematura de cobre en los skarns e intrusivos proporcionalmente (Flores-Bonifacio, 2019).

La mineralización de enargita y tenantina endeble e anómalo enseña la apariencia de arsénico en Toromocho. Los valores más altos de arsénico se hallan ordinariamente por arriba de los $4700 \mathrm{~m}$ y cerca del área exterior (Ver Figura 6-7 y Tabla 1).

\section{RESULTADOS Y DISCUSIÓN}

La mina Toromocho presenta un zonamiento muy complejo donde se tiene minerales muy distintos a otras minas del tipo porfido skarn que no se puede observar macroscópicamente que por ende afecta la metalurgia y el concentrado final, por ese se viene realizando estudios más detallados como difracción de rayos XRD para tener mayor

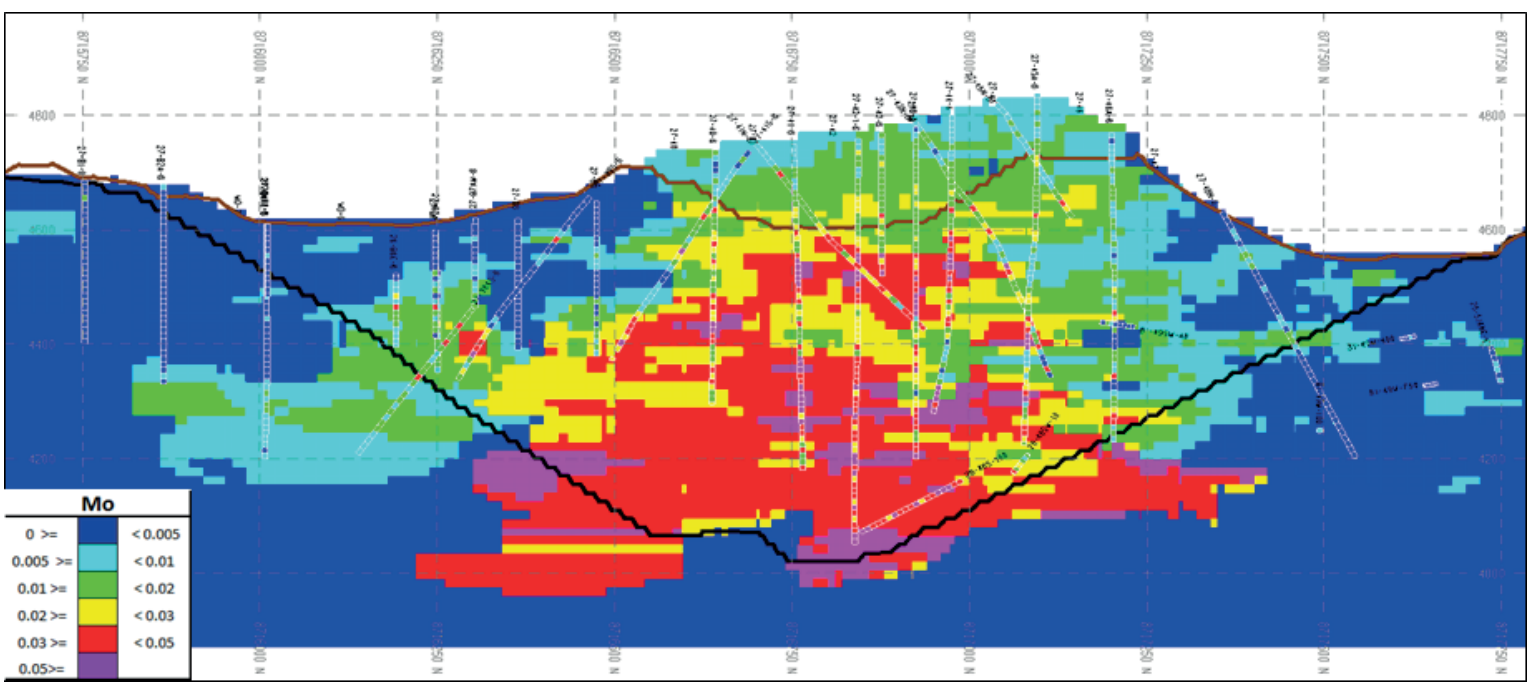

Figura 6. Sección 27 W Molibdeno

Fuente: Minera Chinalco Perú S.A. (2020)
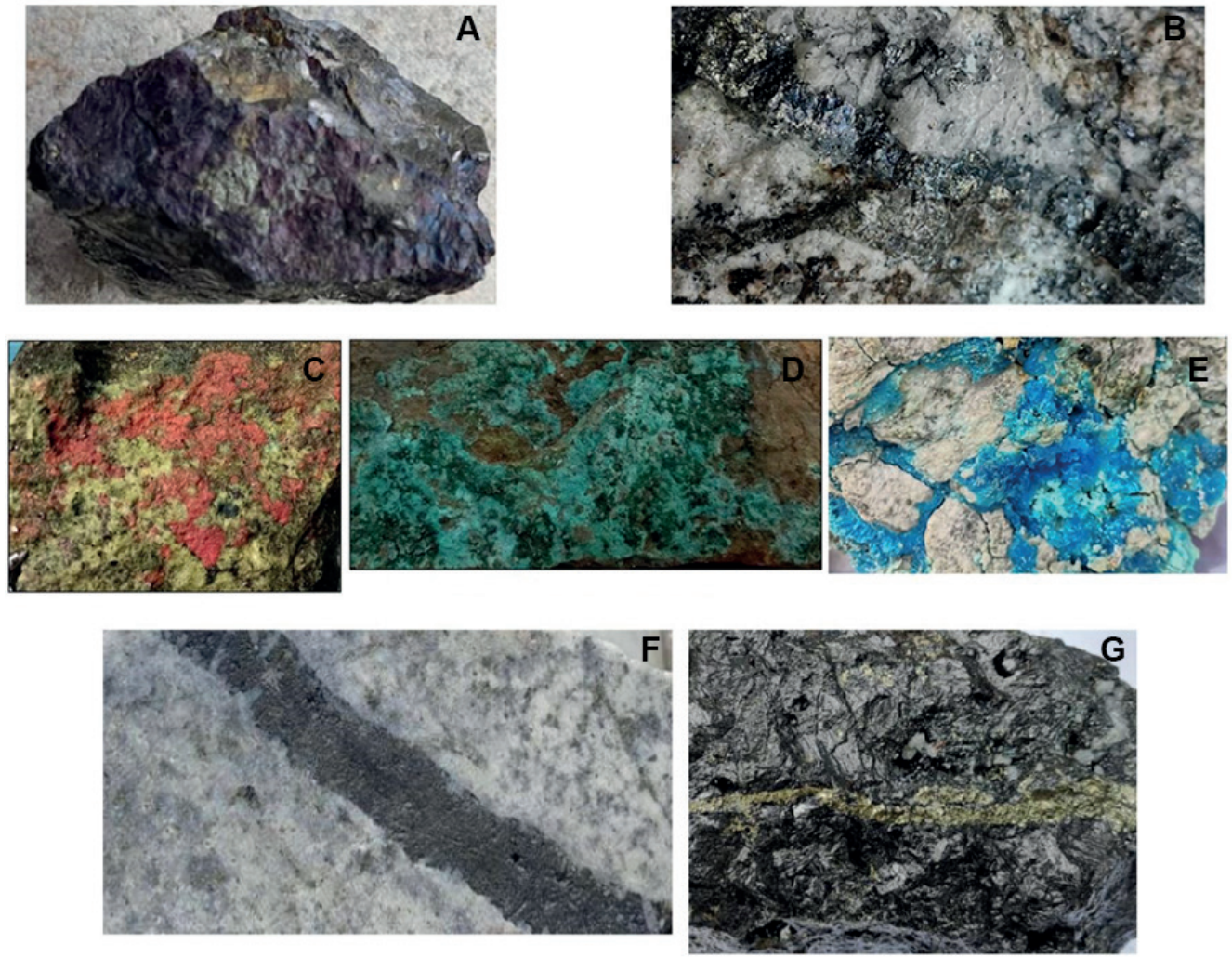

Figura 7. A. Digenite (Cu9S5) B. Covelita (CuS) C. Cuprite $\left(\mathrm{Cu}_{2} \mathrm{O}\right)$ D. Malachite $\left(\mathrm{CuCO}_{3} \cdot \mathrm{Cu}(\mathrm{OH})_{2}\right)$ E. Calcantite (CuSO $\mathrm{CH}_{4} .5 \mathrm{H}_{2} \mathrm{O}$ ) F. Tennantita (Cu12As4S13) G. Enargite (Cu3As4S13)

Fuente: Minera Chinalco Perú S.A. (2020) 


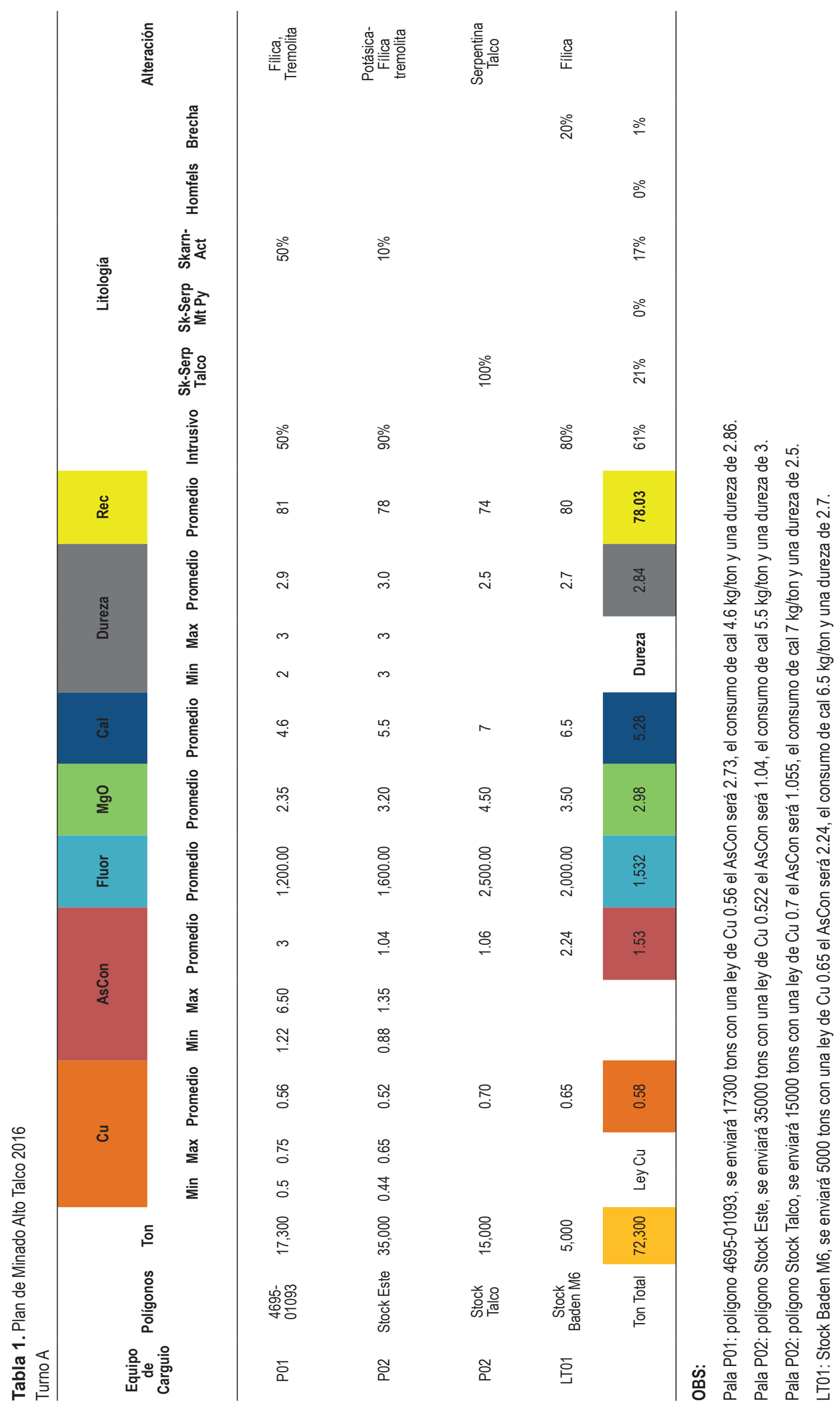


claridad de los minerales que se presenta en la mina y poder tener delimitar el zonamiento mineralógico yacimiento en base al logueo geológico y a los estudios de difracción de rayos $\mathrm{XRD}$.

Los efectos de los estudios de XRD nos permite caracterizar los minerales existentes en el yacimiento, de los minerales contaminantes para poder realizar un buen blending, controlar el talco, fluor, magnesio, zinc etc. en la metalurgia, y poder realizar la dosificación en la planta metalúrgico utilizando el CMD, que nos permite limpiar el concentrado y tener una buena recuperación final.

Los minerales contaminantes en el yacimiento Toromocho consiste en no pasar de los límites máximos permisibles tanto en $500 \mathrm{ppm}$ de fluor, $4 \% \mathrm{MgO}$, zinc $1 \%$, arsénico $0.5 \%$ y la humedad debe ser menos del $15 \%$ todo implica determinar el zonamiento mineralógico para poder realizar el blending.

En el yacimiento Toromocho se tiene que limitar las zonas de minerales contaminantes como es el fluor, $\mathrm{MgO}$, As, zinc para poder realizar un buen blending y tratamiento metalúrgico, poder reducir el contenido del CMC y otros aditivos en la planta comentadora y tener un concentrado bajo parámetros limitados.

\section{CONCLUSIONES}

- Toromocho tiene unas de las mineralogías muy complejas por la cantidad de filosilicatos presentes en el yacimiento, con alteraciones muy variables que conlleva a que el mineral alimentado a la planta de procesos tenga una variabilidad y no sea constante.

- El blending en el yacimiento no solo debe ser por leyes sino también tener en cuenta el porcentaje de talco que debe estar entre $4 \%$ y $5 \%$ y el porcentaje de cada alteración que debe ingresar para obtener para obtener una recuperación mínima de 75\%.

- La técnica de difracción de rayos X a pesar de ser una técnica semicuantitativa, es utilizada por la minera para la caracterización de muestras minerales para la identificación y cuantificación de las especies mineralógicas que pueden estar presentes en las muestras de mineral.

- El talco tiene una relación directa con la recuperación debido a que el talco tiene flotabilidad natural y demás de la influencia que tiene en la estabilidad de burbujas.

- El contenido de altos porcentajes de insolubles en el concentrado final.

- El talco tiende a concentrarse en cada etapa de limpieza del circuito de flotación, el reactivo que se utiliza para poder deprimir el talco es el CMC, pero aun así no es eficiente el cual es adicionado en la etapa cleaner bulk.
- La alteración con mayor contenido de magnetita, pirrotita, pirita, fe, es la magnetita masiva.

- La alteración de magnetita masiva es la que tiene mayores contenidos de cobre soluble en ácido cítrico, cobre soluble en ácido sulfúrico.

- Las litologías que presentan mayores contenidos de cobre soluble en ácido sulfúrico, es el skarn de magnetita masiva, brecha polimictica.

- Las alteraciones que presentan mayor contenido de cobre soluble en ácido sulfúrico son la magnetita masiva, y el skarn de serpentina magnetita.

- Las altas leyes de Molibdeno se encuentran en el intrusivo como también en la brecha polimictica.

- El cobre soluble es mayor en la secuencia sedimentaria compuesto por skarn de serpentina magnetita, hornfles de diópsido, brechas polimicticas.

\section{AGRADECIMIENTOS}

Nuestro agradecimiento a la Universidad Nacional Mayor de San Marcos, nuestra alma mater donde aprendimos a desarrollar los principios de la investigación científica y que en esta ocasión nos permite desarrollar el presente artículo de investigación. A la Unidad de Posgrado de la Facultad de Ingeniería Geológica, Minera, Metalúrgica y Geográfica, a los Docentes.

\section{REFERENCIAS}

Álvarez Angulo, Á. (1999). Experiencias y algunos resultados en exploraciones. https://iimp.org.pe/website2/jueves/ ultimo231/jm20110324 angelalvarez.pdf

Flores-Bonifacio, F. (2019). Implementación de un modelo de bloques en los stocks de mineral en Minera Chinalco Perú [Universidad Nacionaldel Centro del Perú]. repositorio.uncp.edu.pe/bitstream/handle/UNCP/4956/ T010 48517467 T.pdf

Minera Chinalco Perú S.A. (2020). Modificación del estudio de impacto ambiental para le proyecto expansión de la Unidad Minera Toromocho a 170000 TPD. https://www.chinalco. com.pe/meia

Minera Chinalco Perú S.A. Knight Piésold. (2009). Proyecto Toromocho Estudio de Impacto Ambiental (p. 1725). https:// docplayer.es/8450081-Minera-chinalco-peru-s-a-proyectotoromocho-estudio-de-impacto-ambiental-resumenejecutivo.html

Montgomery \& Associates. (2020). Hidrogeología MineraCumplimiento de las Normativas Ambientales. https:// es.elmontgomery.com/?page_id=191

Rondon del Carmen Carlos Eduardo. (2017). Analisis comparativo entre los metodos de explotacion sub level stoping vs corte $y$ relleno convencional en la Mina Morococha. http:// bibliotecas.unsa.edu.pe/handle/UNSA/2569 
